\title{
The thicknesses and inclinations of 71 northern spiral galaxies
}

\author{
Jun Ma ${ }^{1,2,3}$, Qiu-He Peng ${ }^{1}$, and Qiu-Sheng Gu ${ }^{1,2}$ \\ 1 Department of Astronomy, Nanjing University, Nanjing, 210093, China \\ ${ }^{2}$ United Lab. for Optical Astronomy, The Chinese Academy of Sciences, China \\ 3 Shanghai Astronomical Observatory, the Chinese Academy of Sciences, Shanghai 200030, China
}

Received September 23, 1997; accepted January 5, 1998

\begin{abstract}
This paper presents the thicknesses and inclinations (i.e., the angle between the galactic plane and the tangent plane) of 71 northern spiral galaxies. The method for measuring the thickness has been proposed by Peng. It is based on the solution of Poisson's equation for a logarithmic disturbance of density. The inclination is determined by assuming that the pattern of spiral structure is a logarithmic spiral. We find that the thickness is correlated with color and with the $\mathrm{H}_{\alpha}+[\mathrm{NII}]$ equivalent width.
\end{abstract}

Key words: galaxies: spiral; structure; kinematics and dynamics; fundamental parameters

\section{Introduction}

There are two approaches to estimate the thickness of a galactic disc. One, for edge-on spirals, has been suggested by van der Kruit \& Searle (1981a,b, 1982a,b), and is based on surface photometry. The other one, for non-edge-on galaxies, has been proposed by Peng (1988). It is based on the solution of Poisson's equation for a logarithmic disturbance of density. The latter is effective and simple as long as spiral arms are distinguishable on the images.

The inclination of a galaxy (i.e., the angle between the galactic plane and the tangent plane) is difficult to determine. It has been shown by Danver (1942) and Kennicutt \& Hodge (1982) that the spiral arm can be represented by a logarithmic spiral form with constant pitch angle $\mu$. The inclination can be determined by comparing the fitted spiral curve with the image.

In this paper, we present the thicknesses and inclinations for 71 northern spiral galaxies which are selected from more than 600 ones whose images are taken from the Digitized Sky Survey ${ }^{1}$. Most galaxies in this paper

Send offprint requests to: Jun Ma, e-mail: qhpeng@nju.edu.cn

${ }^{1}$ Based on photographic data of the National Geographic Society - Palomar Observatory Sky Survey (NGS-POSS) are grand design spiral ones with Arm Classification $\geq 5$ (Elmegreen \& Elmegreen 1987) except for six spirals. Usually there are two arms for most galaxies, the arm closest to the center is used for measurement.

The structure of this paper is as following: In Sect. 2, we outline the steps how to measure the thickness, determine the inclination, and select the samples. In Sect. 3 , we present the results and discuss errors. We discuss statistical properties in Sect. 4 .

\section{Main steps of the method, and choice of the sample}

The main steps are:

(1). Find the position of the innermost point of a spiral arm and measuring its coordinate $\left(\rho_{0}, \theta_{0}\right)$ relative to the image center and to the major axis.

(2). Vary the value of the inclination $\gamma$ around $\arccos \left(d_{25} / D_{25}\right)$ and fit the spiral arm starting from that innermost point with a logarithmic spiral curve to get its winding parameter $\Lambda$ such that $\mu=\arctan (m / \Lambda)$, where $m$ is the number of arms, with a least square method. $D_{25}$ and $d_{25}$ are taken from the Third Catalog of Bright Galaxies by de Vaucouleurs et al. (1991, RC3). They are the apparent major and minor isophotal diameters measured at or reduced to the surface brightness level $\mu_{B}=25.0$ blue mag per square arc second.

(3). Determine the inclination and the corresponding winding parameter by comparing the fitted spiral curve with the image.

(4). Calculate the thickness of the galactic disk $h$, by

$h=\frac{2 r_{0}}{\sqrt{m^{2}+\Lambda^{2}}}$,

obtained using the Oschin Telescope Palomar Mountain. The NGS-POSS was funded by a grant from the National Geographic Society to the California Institute of Technology. The plates were processed into the present compressed digital form with their permission. The Digitized Sky Survey was produced at the Space Telescope Science Institute under US Government grant NAG W-2166. 
$r_{0}=\frac{\rho_{0} \sqrt{\sin ^{2} \theta_{0}+\cos ^{2} \theta_{0} \cos ^{2} \gamma}}{\cos \gamma}$,

where $m$ is the number of the arms (see Peng 1988).

(5). The thickness of the galactic disk, $H$ in $\mathrm{kpc}$, is $H=h d$,

where $d$ is the distance of the galaxy

$d=\frac{v_{\mathrm{opt}}}{H_{0}}$

where $H_{0}$ is the Hubble constant taken as $75 \mathrm{~km} / \mathrm{s} / \mathrm{Mpc}$, and $v_{\text {opt }}$, the mean heliocentric radial velocity in $\mathrm{km} / \mathrm{s}$ derived from optical observations, is taken from the RC3.

Our statistical sample contains 71 galaxies, selected from more than 600 northern spirals having a determined $(B-V)_{\mathrm{T}}^{0}$ (i.e., the total color index corrected for galactic and internal extinction and redshift) in RC3 according to one single criterion: their images have distinguishable spiral arms. The mean Hubble type indexes $(T)$ of these galaxies are from 2 to 6 , and they have $\log \left(D_{25} / d_{25}\right)$ less than 0.76 .

Our data reduction and analyses were done with $\mathrm{IRAF}^{2}$ installed in the Sun Workstation at the Laboratory of Astronomical Data Analysis of Nanjing University.

\section{Results and errors}

The thicknesses $(H)$, inclinations $(\gamma)$ and winding parameters $(\Lambda)$ of 71 northern spiral galaxies are listed in Tables 1 and 2. Some other parameters are also listed: $m$ is the number of arms, $T$ the mean numerical Hubble stage index, $\mu(=\arctan (m / \Lambda))$ the pitch angle of a spiral arm, $h$ the apparent thickness, $d$ the distance, $H / D_{0}$ the flatness, $D_{0}$ is the isophotal major diameter corrected to the "face-on" $\left(\gamma=0^{\circ}\right)$, and for Galactic extinction, but not for redshift.

The errors arise mainly from: a) the position of the innermost point for the arm; b) the inclination of the galaxy; c) the position of the galactic center. The errors from b) and c), however, can be reduced if we properly modify the grey-scale of the image by using IRAF to obtain the fine structure of the galaxy as deeply as possible. The estimations of error are derived from the formulae given by Peng (1988).

\section{Statistical property}

\subsection{Dependence on color}

Figures 1 and 2 present the correlations between the flatness $\left(H / D_{0}\right)$ and thickness $(H)$ and galaxy color $((B-$

\footnotetext{
${ }^{2}$ IRAF is distributed by the National Optical Astronomy Observatories, which is operated by the Association of Universities for Research in Astronomy, Inc., under cooperative agreement with the National Science Foundation.
}

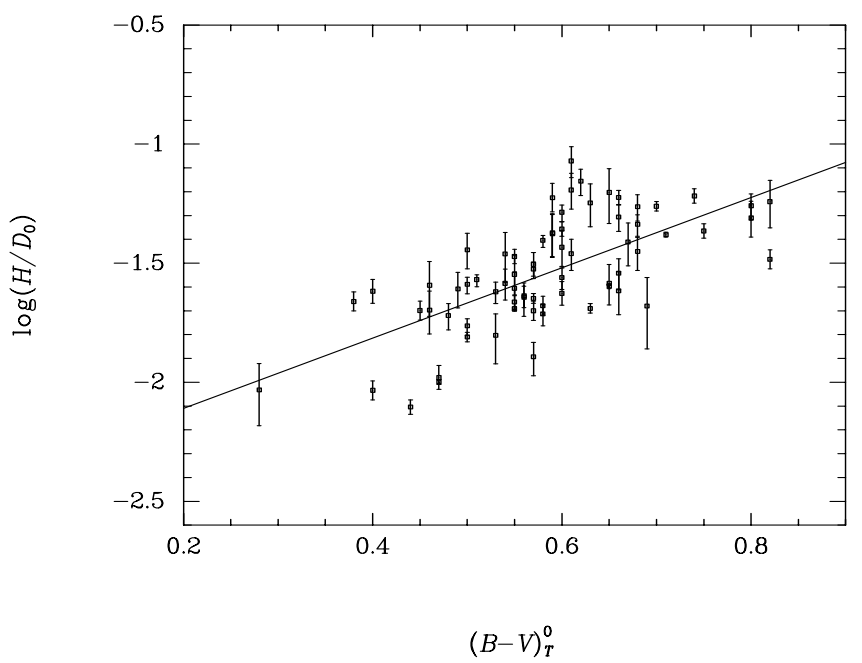

Fig. 1. Flatness of spiral galaxy plotted versus the corrected $B-V$ color

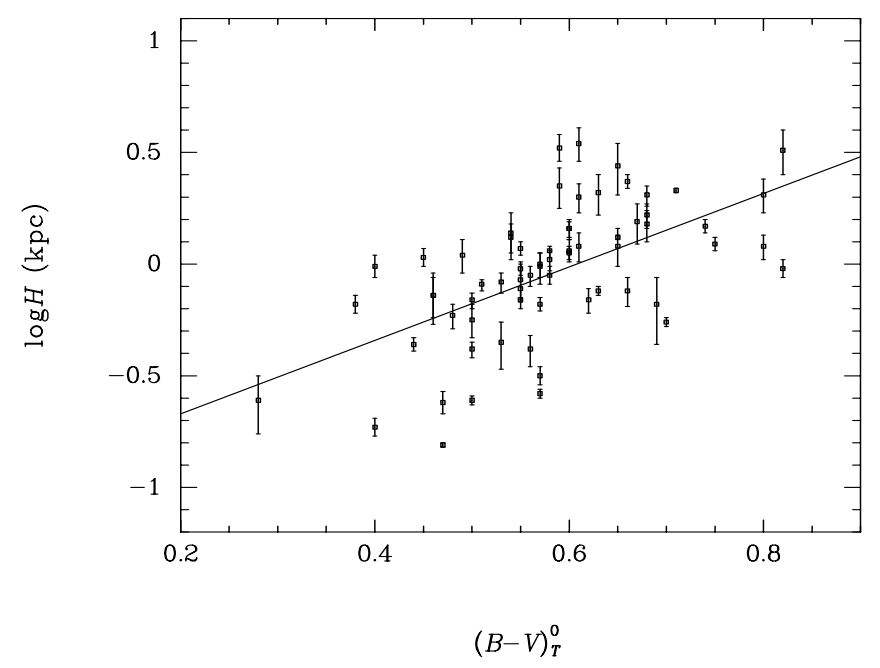

Fig. 2. Thickness of spiral galaxy plotted versus the corrected $B-V$ color

$\left.V)_{\mathrm{T}}^{0}\right)$. The integrated colors of disk galaxies are predominantly influenced by their star formation history (Searle et al. 1973), and the strong correlations observed between color and flatness or thickness point to a coupling between the flatness or thickness and the integrated star formation history of galaxies.

Figure 3 plots flatness as a function of the corrected $U-B$ color $\left((U-B)_{\mathrm{T}}^{0}\right)$, which are taken from RC3, the total $U-B$ color index corrected for differential galactic and internal extinction (to "face-on") and for redshift. The dependence of thickness on $(U-B)_{\mathrm{T}}^{0}$ is illustrated in Fig. 4. 
Table 1. Thicknesses and inclinations of 71 northern spiral galaxies

\begin{tabular}{|c|c|c|c|c|c|c|c|c|c|c|}
\hline PGC & Names & $m$ & $\bar{T}$ & $\begin{array}{c}\gamma \\
\left({ }^{\circ}\right)\end{array}$ & $\overline{\Lambda \pm \mathrm{d} \Lambda / \Lambda}$ & $\begin{array}{c}\mu \\
\left(^{\circ}\right)\end{array}$ & $\begin{array}{c}h \pm \underset{\left({ }^{\prime}\right)}{\mathrm{d} h / h} \\
\end{array}$ & $\begin{array}{c}d \\
(\mathrm{Mpc})\end{array}$ & $\begin{array}{c}H \pm \mathrm{d} H / H \\
(\mathrm{kpc})\end{array}$ & $H / D_{0}$ \\
\hline PGC 1405 & $\begin{array}{ll}\text { NGC } & 91\end{array}$ & 2 & 5.0 & 45.5 & $8.1 \%$ & 23.1 & $0.077 \pm 20.1 \%$ & 68.97 & $1.54 \pm 20.1 \%$ & .039 \\
\hline PGC 2901 & NGC 266 & 2 & 2.0 & 12.2 & $8.91 \pm 23.0 \%$ & 12.7 & $177 \pm 22.6 \%$ & 62.43 & $3.21 \pm 22.6 \%$ & 0.057 \\
\hline PGC 5139 & NGC 514 & 2 & 5.0 & 37.4 & $4.17 \pm 7.7 \%$ & 25.6 & $0.085 \pm 10.0 \%$ & 33.69 & $0.83 \pm 10.0 \%$ & 0.024 \\
\hline PGC 5818 & NGC 598 & 2 & 6.0 & 48.9 & $4.26 \pm 4.3 \%$ & 25.2 & $0.743 \pm 2.8 \%$ & $0.72^{*}$ & $0.16 \pm 2.8 \%$ & 0.010 \\
\hline PGC 6624 & NGC 673 & 2 & 5.0 & 47.1 & $6.47 \pm 13.1 \%$ & 17.2 & $0.054 \pm 16.8 \%$ & 69.88 & $1.10 \pm 16.8 \%$ & 0.025 \\
\hline PGC 6799 & NGC 688 & 2 & 3.2 & 57.9 & $15.06 \pm 25.7 \%$ & 7.6 & $0.087 \pm 23.8 \%$ & 54.81 & $1.39 \pm 23.8 \%$ & 0.035 \\
\hline PGC 7282 & NGC 735 & 2 & 3.0 & 67.1 & $7.33 \pm 18.8 \%$ & 15.3 & $0.066 \pm 15.7 \%$ & 63.19 & $1.21 \pm 15.7 \%$ & 0.035 \\
\hline PGC 10329 & NGC 1073 & 2 & 5.0 & 24.2 & $\pm 12.6 \%$ & 3.6 & $051 \pm 11.3 \%$ & 16.12 & $0.24 \pm 11.3 \%$ & 0.010 \\
\hline PGC 10932 & IC 267 & 2 & 3.0 & 34.1 & $14.39 \pm 13.3 \%$ & 7.9 & $0.119 \pm 13.4 \%$ & 47.69 & $1.65 \pm 13.4 \%$ & 0.055 \\
\hline PGC 13826 & IC 342 & 2 & 6.0 & 12.2 & $9.37 \pm 1.2 \%$ & 12.0 & $0.220 \pm 1.7 \%$ & $3.30^{* *}$ & $0.21 \pm 1.7 \%$ & 0.005 \\
\hline PGC 15212 & $\mathrm{~A} 0423+70$ & 2 & 4.0 & 64.2 & $6.3 \%$ & 23.8 & $0.063 \pm 25.4 \%$ & 39.29 & $0.72 \pm 25.4 \%$ & 0.026 \\
\hline PGC 15867 & NGC 1642 & 2 & 5.0 & 0.0 & $16.6 \%$ & 17.2 & $0.072 \pm 16.4 \%$ & & & 0.037 \\
\hline PGC 17625 & NGC 1961 & 2 & 5.0 & 54.2 & $17.3 \%$ & 5.7 & $0.064 \pm 16.0 \%$ & .11 & $\%$ & 0.013 \\
\hline PGC 20222 & NGC 2339 & 2 & 4.0 & 37.7 & $18.47 \pm 23.4 \%$ & 6.2 & $0.049 \pm 23.3 \%$ & 31.48 & $0.45 \pm 23.3 \%$ & 0.016 \\
\hline PGC 21832 & $\mathrm{~A} 0743+59$ & 2 & 3.0 & 65.7 & $7.46 \pm 20.9 \%$ & 15.0 & $0.089 \pm 20.6 \%$ & 86.77 & $2.25 \pm 20.6 \%$ & 0.042 \\
\hline PGC 22031 & NGC 2441 & 2 & 3.0 & 33.1 & $=7.2 \%$ & 10.4 & $8 \pm 6.5 \%$ & 47.87 & $1.22 \pm 6.5 \%$ & 0.043 \\
\hline PGC 22957 & 2535 & 2 & 5.0 & 30.7 & $.7 \%$ & 22.1 & $062 \pm 11.2 \%$ & 54.39 & $0.98 \pm 11.2 \%$ & 0.024 \\
\hline PGC 23630 & NGC 2582 & 2 & 2.0 & 27.0 & $.3 \%$ & 18.8 & $\pm 13.5 \%$ & 59.48 & $1.19 \pm 13.5 \%$ & 0.055 \\
\hline PGC 24996 & IC 2421 & 2 & 5.0 & 21.1 & $8.7 \%$ & 15.2 & $0.052 \pm 10.1 \%$ & 58.52 & $0.88 \pm 10.1 \%$ & 0.023 \\
\hline PGC 25946 & NGC 2776 & 2 & 5.0 & 32.0 & 8.0 & 14.0 & $0.058 \pm 12.1 \%$ & 34.91 & $0.59 \pm 12.1 \%$ & 0.019 \\
\hline PGC 26666 & NGC 2857 & 2 & 5.0 & 17.0 & $8.42 \pm 6.9 \%$ & 13.4 & $0.047 \pm 8.7 \%$ & 64.85 & $0.89 \pm 8.7 \%$ & 0.021 \\
\hline PGC 27077 & 003 & 2 & 4.0 & 53.4 & $6 \%$ & 12.5 & $\pm 19.1 \%$ & 7.53 & $0.78 \pm 19.1 \%$ & 0.028 \\
\hline PGC 28196 & NGC & 2 & 5.0 & 62.4 & $7 \%$ & 11.4 & $3 \%$ & 63.56 & $3 \%$ & 0.020 \\
\hline PGC 28617 & NGC 3055 & 2 & 5.0 & 46.9 & $16.9 \%$ & 9.7 & $0.077 \pm 17.2 \%$ & 25.07 & $0.56 \pm 17.2 \%$ & 0.036 \\
\hline PGC 28630 & NGC 3031 & 2 & 2.0 & 57.3 & $7.9 \%$ & 13.6 & $0.904 \pm 8.5 \%$ & $3.60^{*}$ & $=8.5 \%$ & 0.033 \\
\hline PGC 30087 & NGC 3184 & 2 & 6.0 & 21.1 & $3.8 \%$ & 19.8 & $0.167 \pm 5.3 \%$ & 5.39 & $0.26 \pm 5.3 \%$ & 0.022 \\
\hline PGC 31883 & $\mathrm{NC}$ & 2 & 5.0 & 54.9 & $\%$ & 14.2 & $3 \pm 5.7 \%$ & 17.29 & $0.82 \pm 5.7 \%$ & 0.027 \\
\hline PGC 31968 & NGC & 2 & 4.0 & 0.0 & 9.0 & 12.4 & $\pm 9.1 \%$ & 7.67 & $0.31 \pm$ & 0.020 \\
\hline PGC 34695 & NGC 3627 & 2 & 3.0 & 62.8 & 5.8 & 19.0 & $\pm 7.3 \%$ & 9.37 & $1.12 \pm 7.3 \%$ & 0.044 \\
\hline PGC 34767 & NGC 3631 & 2 & 5.0 & 22.3 & $.0 \%$ & 17.1 & $0.150 \pm 6.4 \%$ & 15.24 & $0.66 \pm 6.4 \%$ & 0.030 \\
\hline PGC 35105 & $\mathrm{~A} 1122+64$ & 2 & 5.0 & 10.0 & $6.41 \pm 8.0 \%$ & 17.3 & $0.046 \pm 8.8 \%$ & 49.65 & $0.66 \pm 8.8 \%$ & 0.022 \\
\hline PGC 36243 & 3810 & 2 & 5.0 & 49.9 & $6 \%$ & 10.5 & $=7.8 \%$ & 12.77 & $0.42 \pm 7.8 \%$ & 0.026 \\
\hline PGC 36446 & NG & 2 & 4.0 & 30.7 & $3.4 \%$ & 16.2 & $14.4 \%$ & 92.08 & $1.87 \pm 14.4 \%$ & 0.036 \\
\hline PGC 36604 & NGC 3861 & 2 & 3.0 & 56.7 & $11.03 \pm 12.6 \%$ & 10.3 & $0.105 \pm 10.8 \%$ & 67.57 & $2.06 \pm 10.8 \%$ & 0.046 \\
\hline PGC 36902 & NGC 3897 & 2 & 4.0 & 27.0 & $6.91 \pm 7.8 \%$ & 16.1 & $0.046 \pm 11.1 \%$ & 85.79 & $1.15 \pm 11.1 \%$ & 0.024 \\
\hline PGC 37229 & NGC 3938 & 2 & 5.0 & 0.0 & $8.36 \pm 4.1 \%$ & 13.4 & $0.083 \pm 4.0 \%$ & 10.28 & $0.25 \pm 4.0 \%$ & 0.015 \\
\hline PGC 37306 & NGC 3953 & 2 & 4.0 & 59.9 & $14.2 \%$ & 14.5 & $0.198 \pm 14.7 \%$ & 13.16 & $0.76 \pm 14.7 \%$ & 0.029 \\
\hline PGC 37386 & NGC 3963 & 2 & 4.0 & 24.2 & $16.6 \%$ & 14.5 & $0.068 \pm 18.7 \%$ & 42.72 & $0.84 \pm 18.7 \%$ & 0.025 \\
\hline PGC 37617 & NGC 3992 & 2 & 4.0 & 58.5 & $15.69 \pm 36.3 \%$ & 7.3 & $0.162 \pm 33.3 \%$ & 14.12 & $0.67 \pm 33.3 \%$ & 0.021 \\
\hline PGC 38024 & $\mathrm{~A} 1200+41$ & 2 & 4.0 & 54.4 & $6.26 \pm 9.2 \%$ & 17.7 & $0.056 \pm 14.9 \%$ & 81.83 & $1.33 \pm 14.9 \%$ & 0.026 \\
\hline PGC 39028 & NGC 4192 & 2 & 2.0 & 73.6 & $14.35 \pm 24.5 \%$ & 7.9 & $0.421 \pm 20.4 \%$ & & & 0.042 \\
\hline PGC 39483 & IC 3115 & 2 & 6.0 & 35.6 & $13.8 \%$ & 18.8 & $0.086 \pm 13.3 \%$ & & & 0.049 \\
\hline PGC 39600 & NGC 4258 & 2 & 4.0 & 70.6 & $5.91 \pm 9.2 \%$ & 18.7 & $0.627 \pm 7.5 \%$ & 6.40 & $1.17 \pm 7.5 \%$ & 0.034 \\
\hline PGC 39964 & $\mathrm{~A} 1219+41$ & 2 & 3.0 & 31.7 & $12.33 \pm 17.2 \%$ & 9.2 & $0.076 \pm 16.5 \%$ & 92.36 & $2.04 \pm 16.5 \%$ & 0.049 \\
\hline PGC 40001 & NGC 4303 & 2 & 4.0 & 22.0 & $8.35 \pm 8.2 \%$ & 13.5 & $0.111 \pm 8.3 \%$ & 21.43 & $0.69 \pm 8.3 \%$ & 0.017 \\
\hline PGC 40153 & NGC 4321 & 2 & 4.0 & 30.4 & $7.90 \pm 20.1 \%$ & 14.2 & $0.197 \pm 19.0 \%$ & 21.05 & $1.21 \pm 19.0 \%$ & 0.026 \\
\hline
\end{tabular}

\subsection{Dependence on Hubble type}

The tightness of the spiral pattern, in addition to the disk resolution and bulge-to-disk ratio, are the fundamental criteria in Hubble's (1926) classification of spirals. It is interesting to see the dependence of flatness on the Hubble type, which is shown in Fig. 5. The flatness of a spiral galaxy decreases smoothly an average along the Hubble sequence, but the dispersion in flatness among galaxies of the same Hubble type is very large. Figure 6 shows the correlation between thickness and the Hubble sequence. 
Table 2. Thicknesses and inclinations of 71 northern spiral galaxies

\begin{tabular}{|c|c|c|c|c|c|c|c|c|c|c|}
\hline $\mathrm{PGC}$ & Names & $m$ & $T$ & $\begin{array}{c}\gamma \\
\left({ }^{\circ}\right)\end{array}$ & $\overline{\Lambda \pm \mathrm{d} \Lambda / \Lambda}$ & $\begin{array}{c}\mu \\
\left(^{\circ}\right)\end{array}$ & $\begin{array}{c}h \pm \underset{\left({ }^{\prime}\right)}{\mathrm{d} h / h} \\
\end{array}$ & $\begin{array}{c}d \\
(\mathrm{Mpc})\end{array}$ & $\begin{array}{c}H \pm \mathrm{d} H / H \\
(\mathrm{kpc})\end{array}$ & $H / D_{\mathrm{c}}$ \\
\hline$\overline{x C} 40695$ & NGC 4411A & 2 & 5.0 & 29.3 & $9.57 \pm 4.3 \%$ & 11.8 & $0.112 \pm 4.1 \%$ & 16.96 & $0.55 \pm 4.1 \%$ & 0.055 \\
\hline PGC 41812 & NGC 4535 & 2 & 5.0 & 25.9 & $10.60 \pm 9.6 \%$ & 10.7 & $0.137 \pm 10.0 \%$ & 26.31 & $1.05 \pm 10.0 \%$ & 0.019 \\
\hline PGC 42741 & NGC 4639 & 2 & 4.0 & 52.5 & $8.53 \pm 14.2 \%$ & 13.2 & $0.197 \pm 13.0 \%$ & 11.97 & $0.69 \pm 13.0 \%$ & 0.070 \\
\hline PGC 45658 & NGC 5000 & 2 & 4.0 & 31.7 & $8.95 \pm 22.2 \%$ & 12.6 & $0.096 \pm 21.4 \%$ & 75.61 & $2.11 \pm 21.4 \%$ & 0.057 \\
\hline PGC 45948 & NGC 5033 & 2 & 5.0 & 70.1 & $18.32 \pm 24.2 \%$ & 6.2 & $0.215 \pm 21.4 \%$ & 11.48 & $0.72 \pm 21.4 \%$ & 0.020 \\
\hline PGC 47067 & $\mathrm{~A} 1324+20$ & 2 & 4.0 & 37.4 & $9.99 \pm 9.4 \%$ & 11.3 & $0.036 \pm 12.4 \%$ & 95.25 & $1.00 \pm 12.4 \%$ & 0.031 \\
\hline PGC 49514 & NGC 5371 & 2 & 4.0 & 57.4 & $9.48 \pm 27.7 \%$ & 11.9 & $0.274 \pm 26.4 \%$ & 34.33 & $2.74 \pm 26.4 \%$ & 0.063 \\
\hline PGC 49555 & NGC 5364 & 2 & 4.0 & 49.8 & $13.48 \pm 2.6 \%$ & 8.4 & $0.141 \pm 2.3 \%$ & 16.89 & $0.69 \pm 2.3 \%$ & 0.020 \\
\hline PGC 49952 & NGC 5409 & 2 & 3.0 & 41.9 & $23.41 \pm 21.1 \%$ & 4.9 & $0.042 \pm 20.1 \%$ & & & 0.024 \\
\hline PGC 50063 & NGC 5457 & 2 & 6.0 & 41.1 & $8.24 \pm 4.7 \%$ & 13.6 & $0.227 \pm 7.0 \%$ & $6.90^{* * *}$ & $0.44 \pm 7.0 \%$ & 0.008 \\
\hline PGC 52641 & NGC 5740 & 2 & 3.0 & 59.1 & $11.61 \pm 17.9 \%$ & 9.8 & $0.069 \pm 15.9 \%$ & 20.89 & $0.42 \pm 15.9 \%$ & 0.023 \\
\hline PGC 54001 & NGC 5859 & 2 & 4.0 & 71.6 & $6.75 \pm 16.0 \%$ & 16.5 & $0.189 \pm 17.4 \%$ & 62.49 & $3.43 \pm 17.4 \%$ & 0.064 \\
\hline PGC 54849 & NGC 5921 & 2 & 4.0 & 45.6 & $6.05 \pm 8.3 \%$ & 18.3 & $0.253 \pm 7.9 \%$ & 19.43 & $1.43 \pm 7.9 \%$ & 0.052 \\
\hline PGC 55725 & NGC 5985 & 2 & 3.0 & 62.4 & $11.37 \pm 2.7 \%$ & 10.0 & $0.139 \pm 3.2 \%$ & 32.89 & $1.33 \pm 3.2 \%$ & 0.025 \\
\hline PGC 60459 & NGC 6384 & 2 & 4.0 & 53.6 & $9.23 \pm 6.5 \%$ & 12.2 & $0.147 \pm 6.5 \%$ & 22.53 & $0.96 \pm 6.5 \%$ & 0.022 \\
\hline PGC 60635 & IC 1267 & 2 & 3.0 & 57.5 & $6.64 \pm 13.3 \%$ & 16.8 & $0.092 \pm 13.9 \%$ & 124.15 & $3.32 \pm 13.9 \%$ & 0.060 \\
\hline PGC 65001 & NGC 6946 & 2 & 6.0 & 46.7 & $5.54 \pm 6.2 \%$ & 19.8 & $0.154 \pm 8.8 \%$ & $4.20^{* *}$ & $0.19 \pm 8.8 \%$ & 0.009 \\
\hline PGC 65086 & NGC 6951 & 2 & 4.0 & 46.7 & $4.20 \pm 7.9 \%$ & 25.4 & $0.289 \pm 6.0 \%$ & 17.75 & $1.49 \pm 6.0 \%$ & 0.060 \\
\hline PGC 65375 & NGC 6962 & 2 & 2.0 & 55.4 & $11.40 \pm 2.9 \%$ & 10.0 & $0.129 \pm 2.9 \%$ & 56.72 & $2.13 \pm 2.9 \%$ & 0.042 \\
\hline PGC 68110 & $\mathrm{~A} 2206+40$ & 2 & 4.0 & 36.8 & $2.94 \pm 11.9 \%$ & 34.2 & $0.074 \pm 16.1 \%$ & 70.84 & $1.52 \pm 16.1 \%$ & 0.035 \\
\hline PGC 69327 & NGC 7331 & 2 & 3.0 & 72.6 & $9.60 \pm 6.3 \%$ & 11.8 & $0.234 \pm 4.2 \%$ & 11.13 & $0.76 \pm 4.2 \%$ & 0.020 \\
\hline PGC 70144 & A $2255+02$ & 2 & 5.0 & 24.2 & $5.39 \pm 5.4 \%$ & 20.3 & $0.060 \pm 5.9 \%$ & 65.23 & $1.14 \pm 5.9 \%$ & 0.039 \\
\hline PGC 70291 & NGC 7463 & 2 & 3.0 & 76.4 & $30.24 \pm 32.3 \%$ & 3.8 & $0.027 \pm 30.0 \%$ & 30.96 & $0.24 \pm 30.0 \%$ & 0.009 \\
\hline PGC 70419 & NGC 7479 & 2 & 5.0 & 54.7 & $10.60 \pm 7.8 \%$ & 10.7 & $0.255 \pm 7.3 \%$ & 31.92 & $2.37 \pm 7.3 \%$ & 0.060 \\
\hline PGC 71517 & NGC 7677 & 2 & 3.5 & 61.9 & $5.74 \pm 18.2 \%$ & 19.2 & $0.141 \pm 15.5 \%$ & 48.15 & $1.97 \pm 15.5 \%$ & 0.085 \\
\hline
\end{tabular}

* The distance is taken from Faber \& Gallagher (1979).

** The distance is taken from Lang (1983).

*** The distance is taken from Roberts et al. (1975).

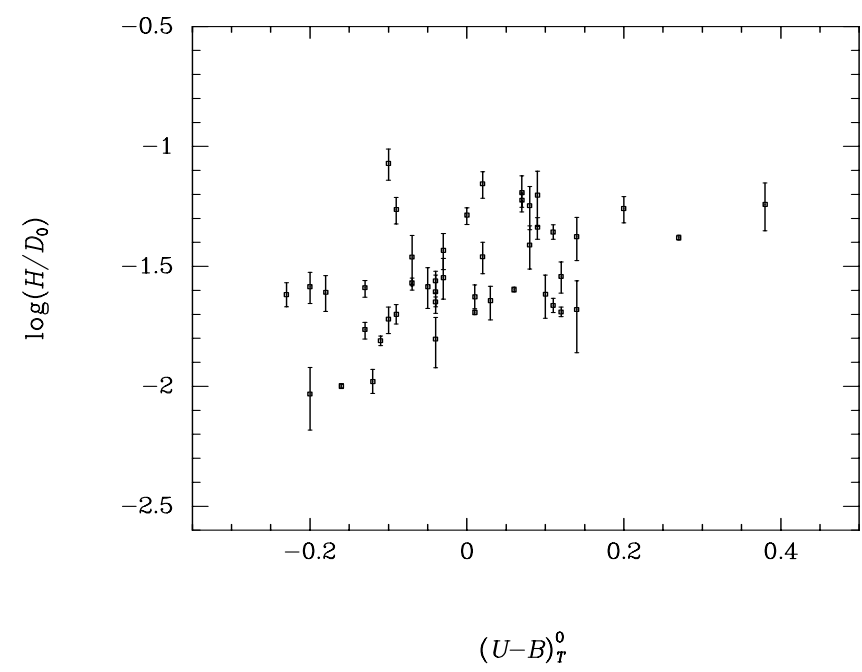

Fig. 3. Flatness of spiral galaxy plotted versus the corrected $U-B$ color

4.3. Correlation between $\mathrm{H}_{\alpha}+[\mathrm{NII}]$ equivalent width and thickness

Kennicutt \& Kent (1983) presented the combined results of photometric and spectrophotometric surveys of

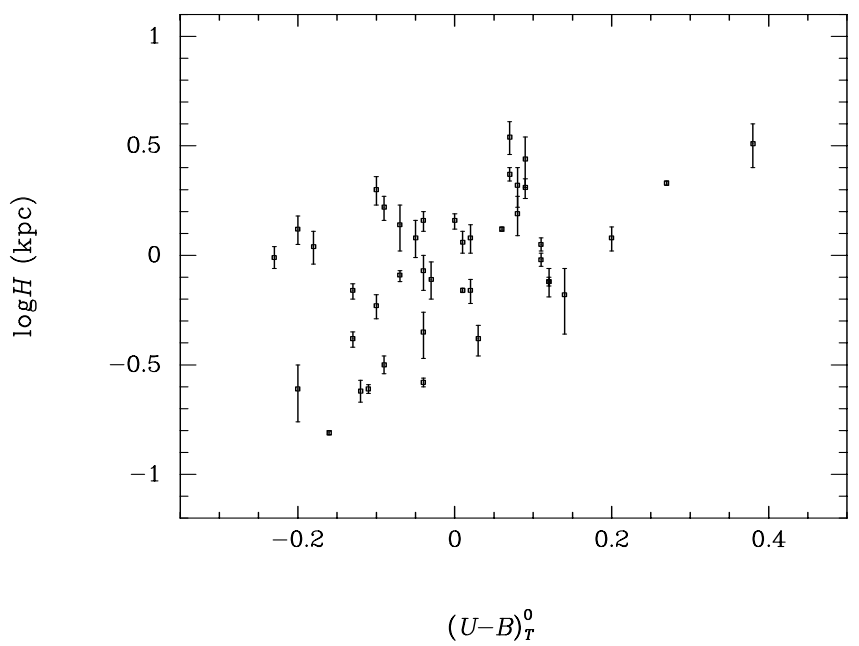

Fig. 4. Thickness of spiral galaxy plotted versus the corrected $U-B$ color

$\mathrm{H}_{\alpha}$ emission for 200 field and Virgo cluster galaxies. Romanishin (1990) published large aperture photometric measurement of $\mathrm{H}_{\alpha}+[\mathrm{NII}]$ emission line strengths of 110 spiral galaxies. We have measured the thicknesses of some 


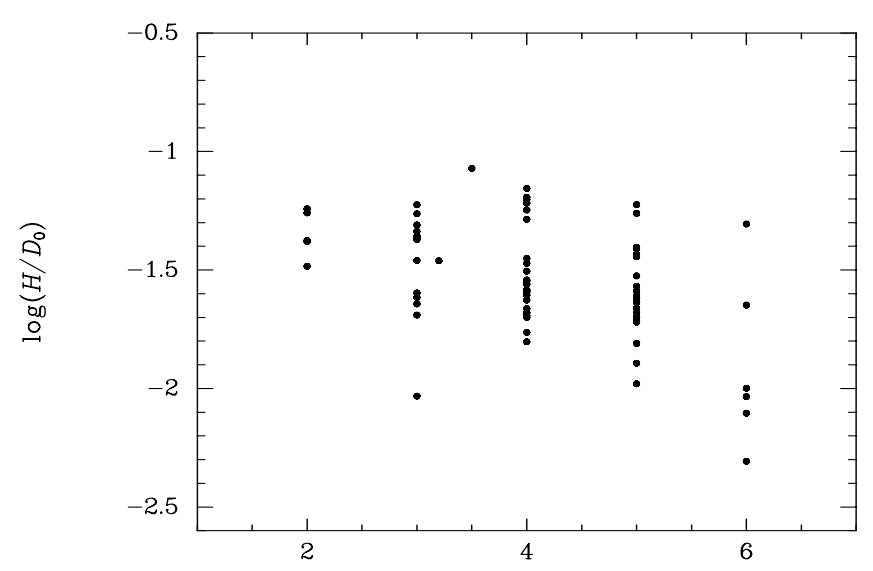

$\mathrm{T}$

Fig. 5. Flatness of spiral galaxy plotted versus the Hubble sequence

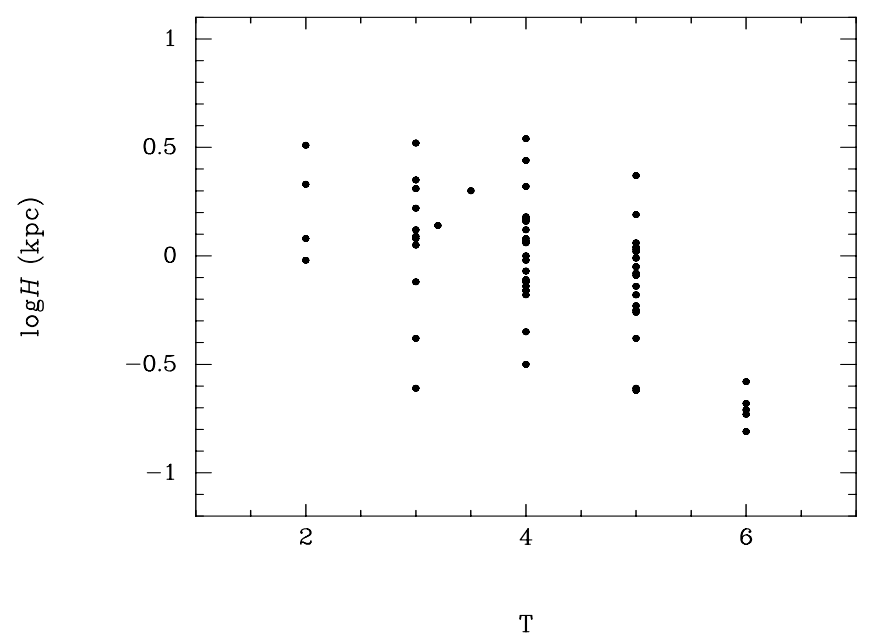

Fig. 6. Thickness of spiral galaxy plotted versus the the Hubble sequence

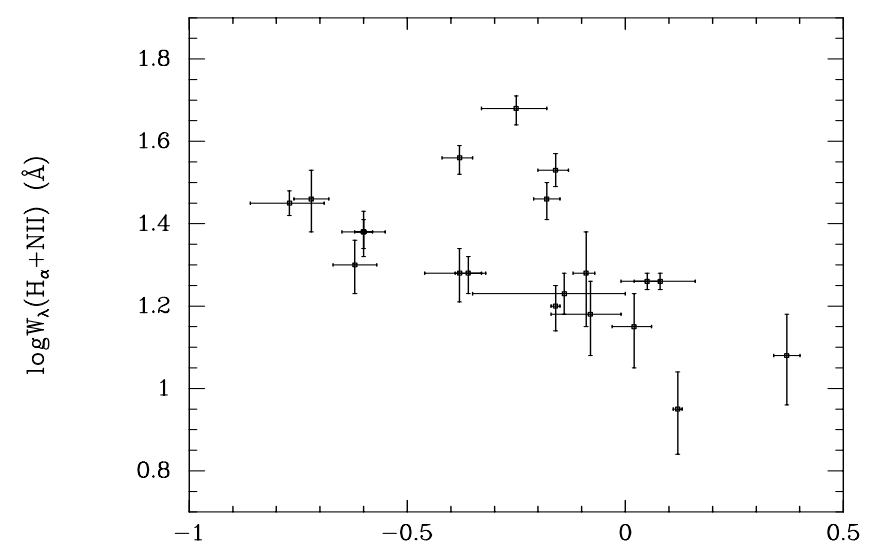

$\log H(\mathrm{kpc})$

Fig. 7. $\mathrm{H}_{\alpha}+[\mathrm{NII}]$ emission line plotted versus the thickness

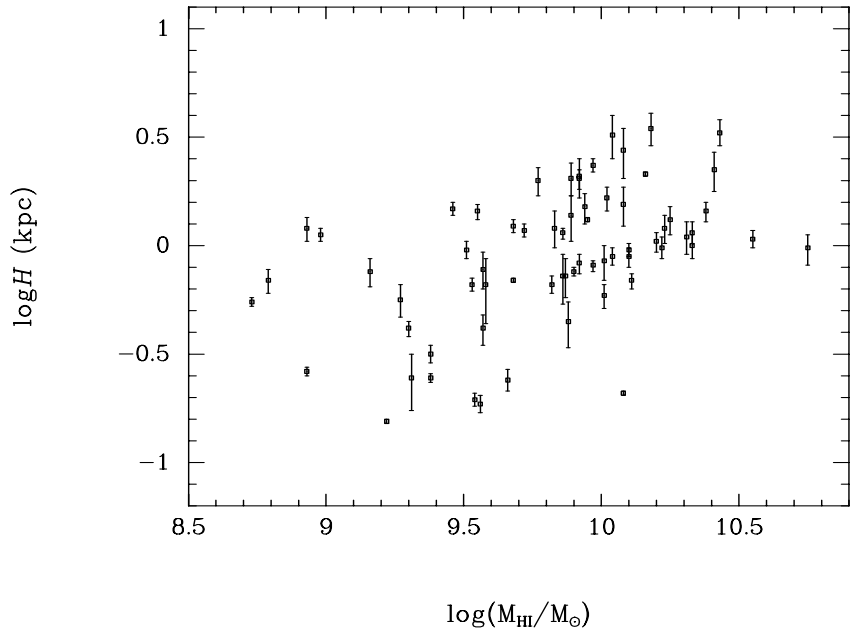

Fig. 8. Thickness of spiral galaxy plotted versus the Neutral hydrogen masses

Table 3. $\mathrm{H}_{\alpha}+[\mathrm{NII}]$ equivalent width and thickness

\begin{tabular}{ccccc}
\hline PGC & Names & $T$ & $H \pm \mathrm{d} H / H(\mathrm{kpc})$ & $\mathrm{EW}(\AA)$ \\
\hline PGC 5974 & NGC 628 & 5.0 & $0.25 \pm 27 \%$ & $24 \pm 2$ \\
PGC 10329 & NGC 1073 & 5.0 & $0.24 \pm 11.3 \%$ & $20 \pm 3$ \\
PGC 28617 & NGC 3055 & 5.0 & $0.56 \pm 17.2 \%$ & $48 \pm 4$ \\
PGC 31883 & NGC 3338 & 5.0 & $0.82 \pm 5.7 \%$ & $19 \pm 5$ \\
PGC 34695 & NGC 3627 & 3.0 & $1.12 \pm 7.3 \%$ & $18 \pm 1$ \\
PGC 34767 & NGC 3631 & 5.0 & $0.66 \pm 6.4 \%$ & $29 \pm 3$ \\
PGC 36243 & NGC 3810 & 5.0 & $0.42 \pm 7.8 \%$ & $36 \pm 3$ \\
PGC 37229 & NGC 3938 & 5.0 & $0.25 \pm 4.0 \%$ & $24 \pm 3$ \\
PGC 37386 & NGC 3963 & 4.0 & $0.84 \pm 18.7 \%$ & $15 \pm 3$ \\
PGC 40001 & NGC 4303 & 4.0 & $0.69 \pm 8.3 \%$ & $34 \pm 3$ \\
PGC 40153 & NGC 4321 & 4.0 & $1.21 \pm 19.0 \%$ & $18 \pm 1$ \\
PGC 41812 & NGC 4535 & 5.0 & $1.05 \pm 10.0 \%$ & $14 \pm 3$ \\
PGC 45948 & NGC 5033 & 5.0 & $0.72 \pm 21.4 \%$ & $17 \pm 2$ \\
PGC 47404 & NGC 5194 & 4.0 & $0.17 \pm 16 \%$ & $28 \pm 2$ \\
PGC 49555 & NGC 5364 & 4.0 & $0.69 \pm 2.3 \%$ & $16 \pm 2$ \\
PGC 50063 & NGC 5457 & 6.0 & $0.44 \pm 7.0 \%$ & $19 \pm 2$ \\
PGC 52641 & NGC 5740 & 3.0 & $0.42 \pm 15.9 \%$ & $19 \pm 3$ \\
PGC 55725 & NGC 5985 & 3.0 & $1.33 \pm 3.2 \%$ & $8.9 \pm 2$ \\
PGC 65001 & NGC 6946 & 6.0 & $0.19 \pm 7.0 \%$ & $29 \pm 5$ \\
PGC 70419 & NGC 7479 & 5.0 & $2.37 \pm 7.3 \%$ & $12 \pm 3$ \\
\hline
\end{tabular}

spirals observed by them. The results are listed in Table 3 . We use the formula (Romanishin 1990):

$\mathrm{EW}$ (Romanishin's) $=1.22 \times \mathrm{EW}(\mathrm{KK}$ 's $)$

Figure 7 shows the correlation of $\mathrm{H}_{\alpha}+[\mathrm{NII}]$ equivalent width with the thickness. There might be a negative correlation between star formation activity and thickness of a galaxy, but the scatter is large and there are only 20 samples. The thicknesses of NGC 628 and NGC 5194 are from Peng's paper (Peng 1988).

Figure 8 plots the relation between thickness and neutral hydrogen masses. The neutral hydrogen mass is derived by de Vaucouleurs et al. (1991, RC3),

$\log \left(M_{\mathrm{HI}} / M_{\odot}\right)=5.696+\left(16.6-m_{21}^{\circ}\right) / 2.5+2.0 \times \log d(5)$ 
where $m_{21}^{\circ}$, from RC3, is the 21-cm emission line magnitude and corrected for self-absorption and $d$, in Mpc, is the distance of a galaxy.

Figure 8 suggests that a thicker galaxy contains more neutral hydrogen, although the dispersion is large.

Acknowledgements. We would like to thank Prof. Jiehao Huang for his help in finishing this paper. We also thank Prof. Jingyao $\mathrm{Hu}$ for his hospitality and discussion at the Xinglong Observational Station of Beijing Observatory, and are grateful to Rui Chen, Zhaohui Ji and Zhaohui Shang for their help. We wish to thank Prof. Zongyun Li for a valuable discussion. This work is supported by the National Nature Science Foundation, the National Grand Project "Climbing Up" of China and the Doctoral Program Foundation of State Education Commission of China.

\section{References}

Danver C.C., 1942, Ann. Obs. Lund. No. 10

de Vaucouleurs G., de Vaucouleurs A., Corwin H.G., Buta B.J., Fouque P., 1991, the Third Reference Catalog of Bright Galaxies. New York: Springer-Verlag
Elmegreen D.M., Elmegreen B.G., 1987, ApJ 314, 3

Faber S.M., Gallagher J.S., 1979, ARA\&A 17, 135

Hubble E.P., 1926, ApJ 64, 321

Kennicutt R.C., Hodge P., 1982, ApJ 253, 101

Kennicutt R.C., Kent S.M., 1983, AJ 88, 1094

Lang K.R., 1980, Astrophysical Formulae. Springer-Verlag Peng Qiu-he, 1988, A\&A 206, 18

Roberts W.W., Roberts M.S., Shu F.H., 1975, ApJ 196, 381

Romanishin W., 1990, AJ 100, 373

Searle L., Sargent W.L.W., Bagnuolo W.G., 1973, ApJ 179, 427

van der Kruit P.C., Searle L., 1981a, A\&A 95, 105

van der Kruit P.C., Searle L., 1981b, A\&A 95, 116

van der Kruit P.C., Searle L., 1982a, A\&A 110, 61

van der Kruit P.C., Searle L., 1982b, A\&A 110, 79 